Article

\title{
Fabrication of Crystalline Microresonators of High Quality Factors with a Controllable Wedge Angle on Lithium Niobate on Insulator
}

\author{
Jianhao Zhang ${ }^{1,2, \dagger}$, Zhiwei Fang ${ }^{3,4, \dagger}$, Jintian Lin ${ }^{1}$, Junxia Zhou ${ }^{3,4}$, Min Wang ${ }^{3,4}{ }^{\oplus}$, \\ Rongbo Wu ${ }^{1,2}$, Renhong Gao ${ }^{1,2}$ and Ya Cheng ${ }^{1,3,4,5, *(\mathbb{D})}$ \\ 1 State Key Laboratory of High Field Laser Physics, Shanghai Institute of Optics and Fine Mechanics, \\ Chinese Academy of Sciences, Shanghai 201800, China \\ 2 Center of Materials Science and Optoelectronics Engineering, University of Chinese Academy of Sciences, \\ Beijing 100049, China \\ 3 State Key Laboratory of Precision Spectroscopy, East China Normal University, Shanghai 200062, China \\ 4 The Extreme Optoelectromechanics Laboratory, School of Physics and Materials Science, \\ East China Normal University, Shanghai 200241, China \\ 5 Collaborative Innovation Center of Extreme Optics, Shanxi University, Taiyuan 030006, China \\ * Correspondence: ya.cheng@siom.ac.cn \\ + These authors contributed equally to this work.
}

Received: 24 July 2019; Accepted: 26 August 2019; Published: 29 August 2019

check for updates

\begin{abstract}
We report the fabrication of crystalline microresonators of high quality (Q) factors with a controllable wedge angle on lithium niobate on insulator (LNOI). Our technique relies on a femtosecond laser assisted chemo-mechanical polish, which allows us to achieve ultrahigh surface smoothness as critically demanded by high $\mathrm{Q}$ microresonator applications. We show that by refining the polish parameters, $\mathrm{Q}$ factors as high as $4.7 \times 10^{7}$ can be obtained and the wedge angle of the LNOI can be continuously tuned from $9^{\circ}$ to $51^{\circ}$.
\end{abstract}

Keywords: lithium niobate microdisk resonator; controllable wedge angle; high quality factors; chemo-mechanical polish

\section{Introduction}

Whispering gallery microresonators (WGM) are miniaturized optical cavities where light waves can travel along the circular periphery with extremely low propagation losses by means of total internal reflection at the glass-air interface. So far, WGMs have been realized in various kinds of transparent materials such as liquids, polymers, glasses, semiconductors, and dielectric crystals [1-3]. The physical and/or optical functionalities demonstrated with WGMs include lasing, filtering, nonlinear wavelength conversion, optomechanics, and cavity electrodynamics, to name only a few [4-9]. Currently, it is of high interest to realize on-chip integration of the WGMs with other photonic micro- and nanostructures. The material platforms suitable for photonic integration application include traditional semiconductors for their fabrication compatibility with the complementary metal oxide semiconductor(CMOS) approach and the emerging lithium niobate on insulator (LNOI), thanks to the rapid development of innovative solutions to produce high quality LNOI photonic structures [10-15].

Very recently, we have demonstrated the fabrication of high Q LNOI microdisk resonators using chemo-mechanical polish lithography [16-18]. The $Q$ factor was measured as $1.46 \times 10^{7}$ at a wavelength of around $773 \mathrm{~nm}$. As a typical feature of chemo-mechanical polish, the fabricated microdisk showed an extended wedge at the rim of a wedge angle of $\sim 9.5^{\circ}$. High $Q$ microresonators have been used to demonstrate electric tunable optomechanics [19]. Nevertheless, for most WGM applications, it is 
always desirable to improve the $\mathrm{Q}$ factor and to have the capability of controlling the dispersion property with a tunable wedge angle. This provides a strong incentive for us to carry out systematic investigations into the optimizations of the $Q$ factor and wedge angle by refining the fabrication parameters, which will be shown in detail below.

\section{Experimental Details}

In our investigation, the LNOI microdisks with variable diameters and wedge angles were produced on a commercially available X-cut LNOI wafer (NANOLN, Jinan Jingzheng Electronics Co. Ltd. Jinan, China). The lithium niobate (LN) thin film with a thickness of $900 \mathrm{~nm}$ was bonded to a $2 \mu$ m-thick $\mathrm{SiO}_{2}$ layer supported by a $500-\mu \mathrm{m}$-thick $\mathrm{LN}$ substrate.

As shown in Figure 1, the fabrication process includes four steps as briefly described as follows. (1) Deposition of a thin layer of chromium (Cr) with a thickness of $600 \mathrm{~nm}$ on the surface of the LNOI by magnetron sputtering. (2) Patterning of $\mathrm{Cr}$ film using femtosecond laser ablation. (3) Removing the uncovered LNOI by chemo-mechanical polishing. (4) Chemical wet etching to remove the Cr mask. The pulse energy of the femtosecond laser was carefully adjusted to enable the complete removal of the Cr film without damaging the underneath LNOI because the damage threshold of the LNOI is significantly higher than $\mathrm{Cr}$ under the irradiation of femtosecond laser pulses. The details in the femtosecond laser ablation are provided herein. We used a femtosecond laser with a center wavelength of $1030 \mathrm{~nm}$ and a pulse width of $170 \mathrm{fs}$ (PHAROS, LIGHT CONVERSION) for patterning the Cr film at the average power of $0.1 \mathrm{~mW}$. A 100× objective lens (M Plan Apo NIR, Mitutoyo Corporation, NA0.7, Kawasaki, Kanagawa, Japan) was used to produce a tightly focused spot of $\sim 1 \mu \mathrm{m}$ in diameter.

In the CM polishing process using a wafer-polishing machine (NUIPOL802, Kejing, Inc.Hefei, China), the wedge angle can be controlled by changing the duration of the polishing process. More details of CM polishing can be found elsewhere [16,17].
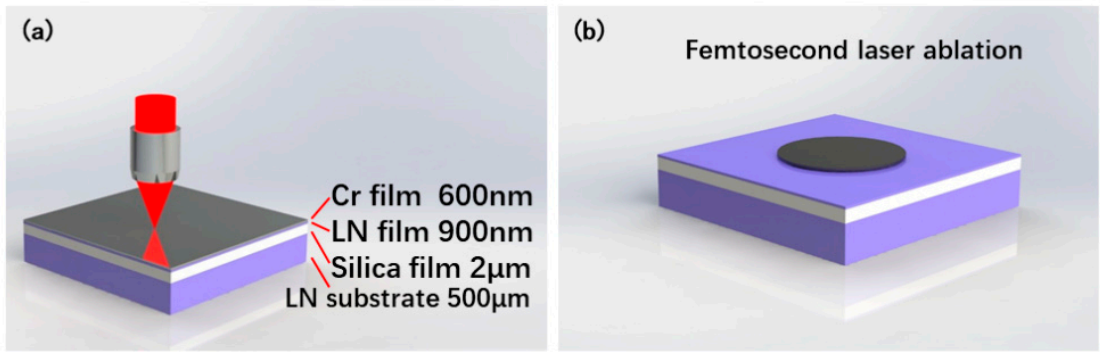

(c)

Chemo-mechanical polish(CMP)

(d)

$\mathrm{Cr}$ and silica removal
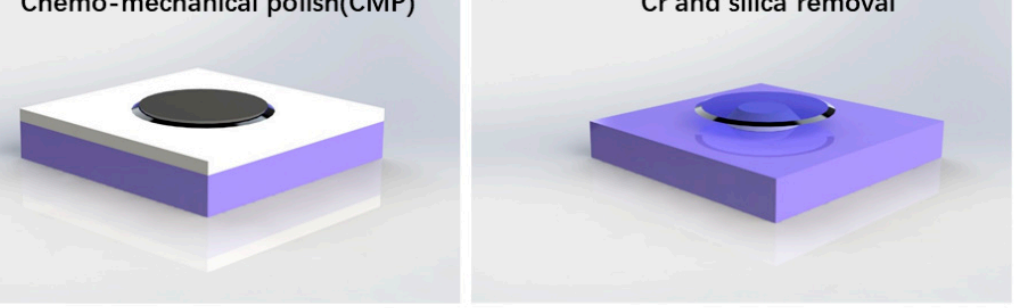

Figure 1. (a-d) Flowchart of fabricating an on-chip lithium niobate microdisk resonator.

\section{Results and Discussion}

Figure 2a shows a LNOI microdisk fabricated using a $\mathrm{SiO}_{2}$ slurry with a particle size of $\sim 20 \mathrm{~nm}$. The close-up view of the area indicated by the red rectangular frame is shown in Figure $2 b$, presented $a$ smooth surface morphology in the optical micrograph. Figure $2 \mathrm{c}$ presents the atomic force microscope (AFM) image of the polished surface by which an ultralow surface roughness of $\mathrm{Rq} \sim 0.115 \mathrm{~nm}$ could be determined. The surface roughness was significantly lower than that reported in our previous work ( $\mathrm{Rq} \sim 0.452 \mathrm{~nm}$ ) due to the fact that the slurry with a particle size of $\sim 60 \mathrm{~nm}$ was used previously [16]. 
Actually, we systematically examined the surface roughness with the particle size and found that a particle size of $\sim 20 \mathrm{~nm}$ could give us the optimum surface roughness and a reasonable polish duration. Figure $2 \mathrm{~d}$ exhibits all the microresonators with different diameters ranging from $55 \mu \mathrm{m}$ to $205 \mu \mathrm{m}$. All of the microresonators showed a highly reproducible smooth surface morphology.
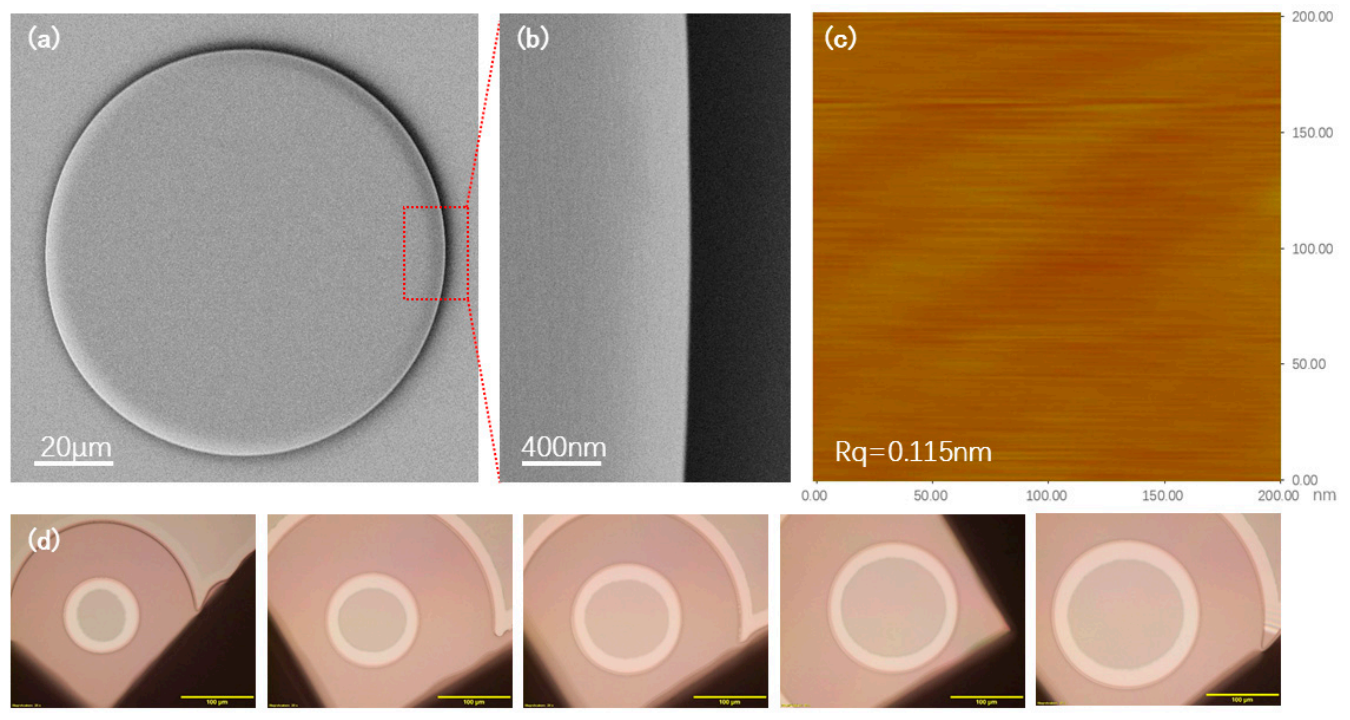

Figure 2. (a) Top view scanning electron microscope (SEM) image of a fabricated LN microdisk resonator. (b) Close up view SEM image of the area indicated by the red box in (a). (c) Atomic force microscope (AFM) image of the microdisk wedge. (d) Optical microscope image of the microdisk resonator with different diameters $(55 \mu \mathrm{m}, 85 \mu \mathrm{m}, 105 \mu \mathrm{m}, 135 \mu \mathrm{m}, 155 \mu \mathrm{m}, 185 \mu \mathrm{m}$, and $205 \mu \mathrm{m})$.

With a surface roughness to the order of $\mathrm{Ra} \sim 0.115$, the major source of optical loss in the microresonator is radiative bending loss, which can be reduced by increasing the diameters of the microdisks. To confirm this, we measured the $Q$ factor of each microresonator in Figure $2 \mathrm{~d}$. The results are presented in Table 1 and Figure 3a. Indeed, it can be seen that the $Q$ factor does undergo an increase for the microdisks with diameters of $55 \mu \mathrm{m}, 85 \mu \mathrm{m}$, and $105 \mu \mathrm{m}$. However, after the $\mathrm{Q}$ factor reached its peak, which was $4.7 \times 10^{7}$ obtained with the microdisk of a diameter of $105 \mu \mathrm{m}$, the $\mathrm{Q}$ factor decreased with the further increase of the diameter of the microdisk. It should be noted that to avoid the fluctuations of the measured $Q$ factors resulting from the inconsistency in the fabrication process, we fabricated three samples for each diameter, and then made three measurements accordingly. The general trend in Figure $3 \mathrm{a}$ is therefore a reliable feature of the $\mathrm{Q}$ factor dependence on the microdisk diameter.

Table 1. $\mathrm{Q}$ factors of the microdisks with different diameters.

\begin{tabular}{cccc}
\hline Diameter & Q-Factors (Sample i) & Q-Factors (Sample ii) & Q-Factors (Sample iii) \\
\hline $55 \mu \mathrm{m}$ & $2.15 \times 10^{7}$ & $2.26 \times 10^{7}$ & $2.74 \times 10^{7}$ \\
$85 \mu \mathrm{m}$ & $2.17 \times 10^{7}$ & $2.51 \times 10^{7}$ & $3.73 \times 10^{7}$ \\
$105 \mu \mathrm{m}$ & $3.22 \times 10^{7}$ & $4.16 \times 10^{7}$ & $4.70 \times 10^{7}$ \\
$130 \mu \mathrm{m}$ & $2.94 \times 10^{7}$ & $3.32 \times 10^{7}$ & $3.30 \times 10^{7}$ \\
$155 \mu \mathrm{m}$ & $1.95 \times 10^{7}$ & $2.76 \times 10^{7}$ & $3.16 \times 10^{7}$ \\
$180 \mu \mathrm{m}$ & $2.03 \times 10^{7}$ & $2.07 \times 10^{7}$ & $3.12 \times 10^{7}$ \\
$205 \mu \mathrm{m}$ & $1.78 \times 10^{7}$ & $1.92 \times 10^{7}$ & $2.67 \times 10^{7}$ \\
\hline
\end{tabular}



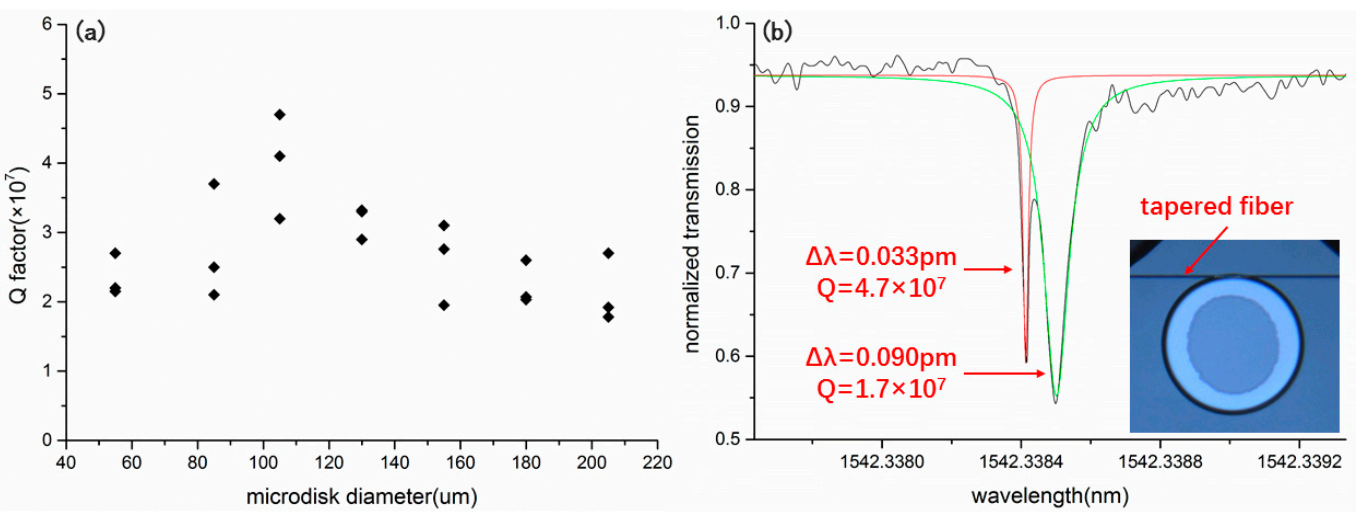

Figure 3. (a) Measured $Q$ factors of the mcirodisks of different diameters. (b) The Lorentz fitting (red curve) of a splitting mode in the microdisk with a diameter of $105 \mu \mathrm{m}$ revealed a $\mathrm{Q}$ factor of $4.7 \times 10^{7}$.

We attempted to understand the unexpected trend of the decreasing $Q$ factor with the increasing microdisk diameter after the diameter of the microdisk reached $105 \mu \mathrm{m}$. To do so, we conducted simulations of the light fields (electric field) in the microdisks with different diameters but with the same wedge angle of $10^{\circ}$, and the thickness of the LN thin film was $900 \mathrm{~nm}$. Note that we did not calculate the $\mathrm{Q}$ factors of the microdisks with different diameters as many key parameters (such as the exact diameter, thickness, and surface roundness of each microdisk) cannot be measured with absolute precision. The simulation results in Figure 4 revealed that with the increase in the diameter of the microdisk, the fundamental (Figure $4 \mathrm{c}-\mathrm{e}$ ) and high-order (Figure $4 \mathrm{f}-\mathrm{h}$ ) modes tended to penetrate more deeply toward the center of the microdisk. It is highly likely that the tail of the fundamental as well as high-order modes in the microdisks of large diameters may scratch the underneath pedestal supporting the freestanding microdisk, giving rise to a decrease in the $Q$ factors. In our work, the diameter of the fused silica pedestal was controlled as $\sim 20 \mu \mathrm{m}$ less than that of the microdisk, otherwise the freestanding microdisk may collapse in the chemical wet etching to partially remove the fused silica. For this reason, some fused silica may remain unremoved on the backside of the microdisk during the short period of chemical wet etching, giving rise to a slight scattering loss and in turn, a slight decrease in the $\mathrm{Q}$ factors.
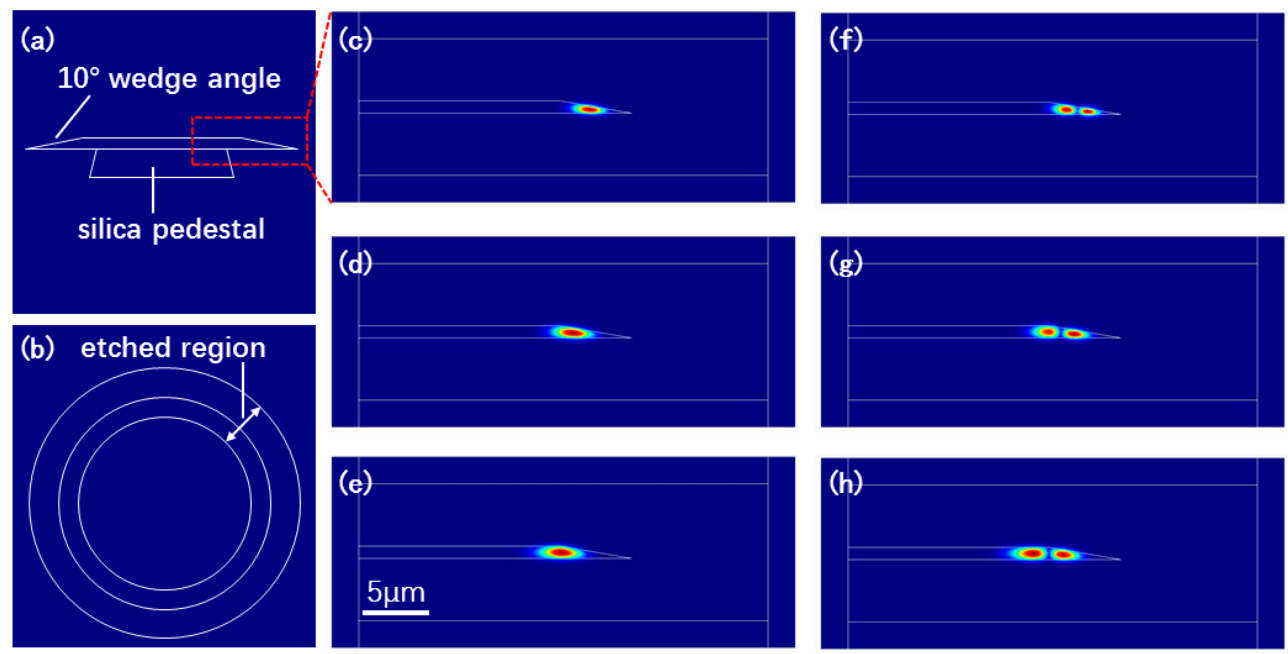

Figure 4. Side view (a) and top view (b) of the structure of the simulated microdisk resonator. Electric field of the fundamental modes in the microdisk resonator with a diameter of (c) $50 \mu \mathrm{m},(\mathbf{d}) 100 \mu \mathrm{m}$, and (e) $200 \mu \mathrm{m}$. Electric field of second-order modes in the microdisk resonator with a diameter of (f) $50 \mu \mathrm{m},(\mathrm{g}) 100 \mu \mathrm{m}$, and (h) $200 \mu \mathrm{m}$. The modes show a general feature where the mode size becomes larger with the increasing order of mode. 
We also investigated the dependence of the wedge angle of the microdisk on the polish duration. In general, the longer the polishing process, the larger the wedge angle of the microdisk. Figure 5a-f show the microdisks with a diameter of $85 \mu \mathrm{m}$ obtained with polish durations of $12 \mathrm{~min}, 16 \mathrm{~min}, 18 \mathrm{~min}$, $24 \mathrm{~min}, 30 \mathrm{~min}, 42 \mathrm{~min}$, and $60 \mathrm{~min}$, respectively. Accordingly, the wedge angles of the microdisks in Figure $5 \mathrm{a}-\mathrm{f}$ were measured as $9^{\circ}, 14^{\circ}, 22^{\circ}, 30^{\circ}, 40^{\circ}$, and $51^{\circ}$, respectively. The measured $\mathrm{Q}$ factors of the microdisks with different wedge angles are provided in Figure $5 \mathrm{~g}$. The $\mathrm{Q}$ factors showed almost no dependence on the wedge angle, and were significantly higher than $1 \times 10^{7}$ at all wedge angles.
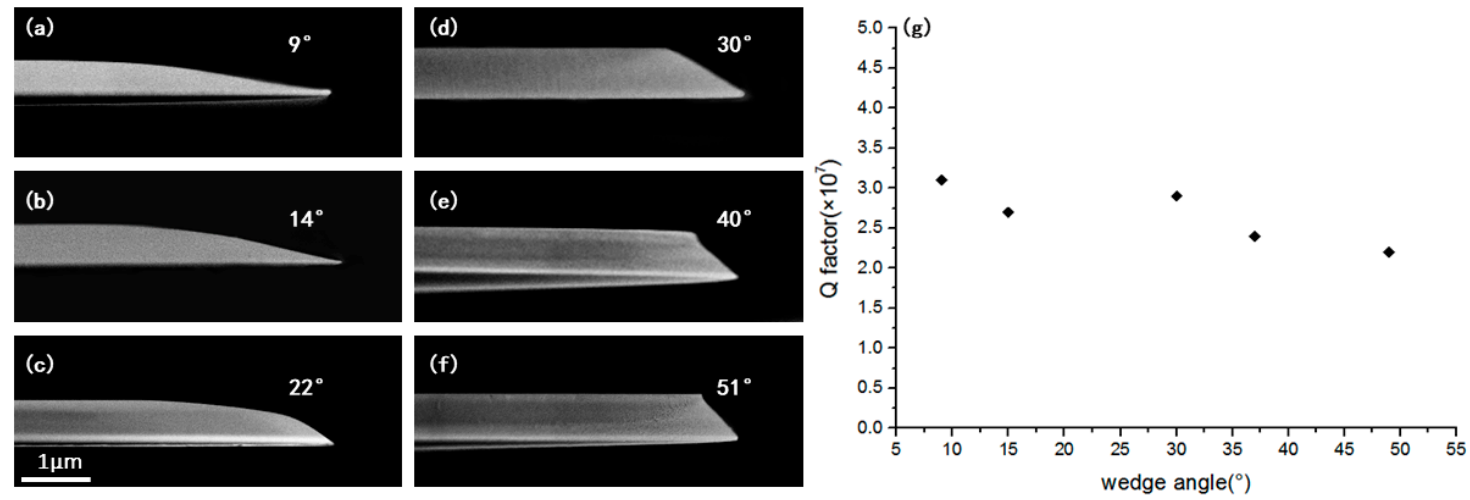

Figure 5. (a-f) Side view scanning electron microscope (SEM) image of the fabricated LN microdisks with different wedge angles of $9^{\circ}, 14^{\circ}, 22^{\circ}, 30^{\circ}, 40^{\circ}$, and $51^{\circ}$, respectively. (g) Q factors of the microdisks with different wedge angles.

Finally, we noticed that from the side-view image in Figure 6, the microdisks of large wedge angles obtained with long polish durations appeared to be composed of a microdisk with a vertical sidewall (see region I in Figure 6) stacked on top of another microdisk with an extended wedge (see region II in Figure 6). The thicknesses of region I and II were measured as $280 \mathrm{~nm}$ and $420 \mathrm{~nm}$, respectively. In general, this should be caused by the mechanical interaction of the LNOI microdisks with the polishing cloth, whereas understanding the underlying details requires further investigations in a systematic manner. Nevertheless, such complex geometry may provide innovative opportunities of controlling light fields in the LNOI microdisk resonators.

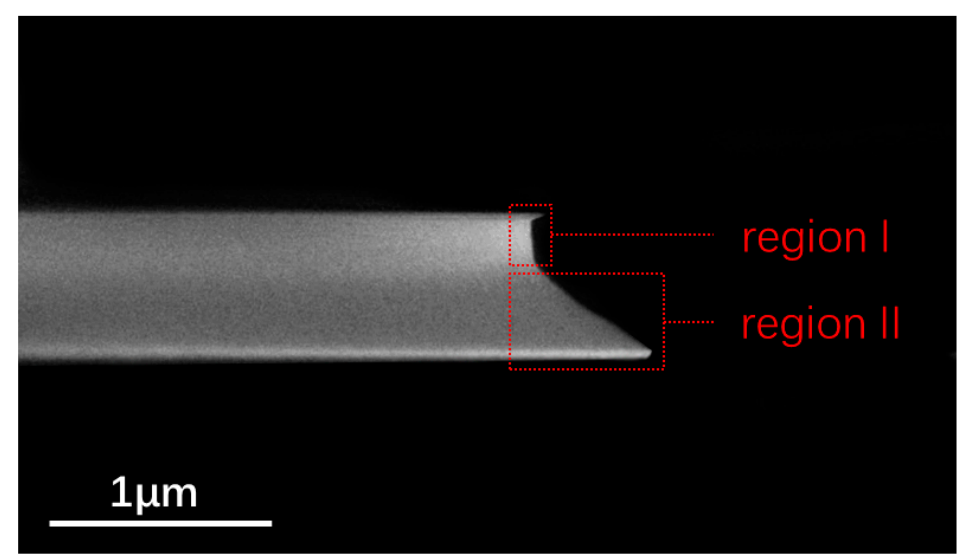

Figure 6. Side view scanning electron microscope (SEM) image of a fabricated LN microdisk resonator with a long polish duration of $90 \mathrm{~min}$.

\section{Conclusions}

To conclude, we demonstrated the optimization of the $\mathrm{Q}$ factor and wedge angle of LNOI microdisk resonators fabricated by femtosecond laser assisted chemo-mechanical polish. We achieved, to the 
best of our knowledge, a record-high $Q$ factor of $4.7 \times 10^{7}$ by improving the surface smoothness and optimizing the diameter of the LNOI microdisk resonator. We showed that the wedge angle of the LNOI could be continuously tuned from $9^{\circ}$ to $51^{\circ}$ without spoiling the $\mathrm{Q}$ factor, which is critical for nonlinear optical applications as the dispersion curve in the microdisks is a function of the wedge angle. Thus, our results have important implications for applications ranging from classical and non-classical light sources to optical comb generation and optomechanics.

Author Contributions: Conceptualization, Y.C.; Methodology, Y.C.; Software, J.Z. (Jianhao Zhang); Validation, J.Z. (Jianhao Zhang) and Z.F.; Formal analysis, J.Z. (Jianhao Zhang); Investigation, J.Z. (Jianhao Zhang), J.L., and Z.F.; Resources, J.Z. (Jianhao Zhang), M.W., Z.F., R.W., J.Z. (Junxia Zhou), R.G., and J.L.; Data curation, Z.F. and M.W.; Writing-original draft preparation, Y.C. and J.Z. (Jianhao Zhang); Writing-review and editing, Y.C., J.Z. (Jianhao Zhang), M.W., J.L. R.G., and R.W.; Visualization, J.Z. (Jianhao Zhang), and M.W.; Supervision, Y.C.; Funding acquisition, Y.C. and J.L.

Funding: This research was funded by the National Natural Science Foundation of China (Grant Nos. 11734009, 11874375, 61590934, 11874154, 61505231, 11604351, 11674340, 61575211, 61675220, and 61761136006), the Strategic Priority Research Program of Chinese Academy of Sciences (Grant No. XDB16030300), the Key Research Program of Frontier Sciences, the Chinese Academy of Sciences (Grant No. QYZDJ-SSW-SLH010), the Project of Shanghai Committee of Science and Technology (Grant No. 17JC1400400), the Shanghai Rising-Star Program (Grant No. 17QA1404600), the National Key R\&D Program of China (Grant No. 2018YFB0504400), and the Shanghai Pujiang Program (Grant No. 18PJ1403300).

Conflicts of Interest: The authors declare no conflict of interest.

\section{References}

1. Vahala, K.J. Optical microcavities. Nature 2003, 424, 839-846. [CrossRef] [PubMed]

2. Jiang, X.F.; Zou, C.L.; Wang, L.; Gong, Q.; Xiao, Y.-F. Whispering-gallery microcavities with unidirectional laser emission. Laser Photonics Rev. 2016, 10, 40-61. [CrossRef]

3. Lin, G.; Chembo, Y.K. Monolithic total internal reflection resonators for applications in photonics. Opt. Mater. X 2019, 2, 100017. [CrossRef]

4. Spillane, S.M.; Kippenberg, T.J.; Vahala, K.J. Ultralow-threshold Raman laser using a spherical dielectric microcavity. Nature 2002, 415, 621-623. [CrossRef] [PubMed]

5. Feng, S.; Lei, T.; Chen, H.; Luo, X.; Poon, A.W. Silicon photonics: From a microresonator perspective. Laser Photonics Rev. 2012, 6, 145-177. [CrossRef]

6. Kippenberg, T.J.; Vahala, K.J. Cavity optomechanics: Back-action at the mesoscale. Science 2008, 321, 1172-1176. [CrossRef] [PubMed]

7. Foreman, M.R.; Swaim, J.D.; Vollmer, F. Whispering gallery mode sensors. Adv. Opt. Photonics 2015, 7, 168-240. [CrossRef] [PubMed]

8. Lin, G.; Coillet, A.; Chembo, Y.K. Nonlinear photonics with high-Q whispering-gallery-mode resonators. Adv. Opt. Photonics 2017, 9, 828-890. [CrossRef]

9. Spillane, S.M.; Kippenberg, T.J.; Painter, O.J.; Vahala, K.J. Ideality in a fiber-taper-coupled microresonator system for application to cavity quantum electrodynamics. Phys. Rev. Lett. 2003, 91, 043902. [CrossRef] [PubMed]

10. Lin, J.; Xu, Y.; Fang, Z.; Wang, M.; Song, J.; Wang, N.; Qiao, L.; Fang, W.; Cheng, Y. Fabrication of high-Q lithium niobate microresonators using femtosecond laser micromachining. Sci. Rep. 2015, 5, 8072. [CrossRef] [PubMed]

11. Wang, J.; Bo, F.; Wan, S.; Li, W.; Gao, F.; Li, J.; Zhang, G.; Xu, J. High-Q lithium niobate microdisk resonators on a chip for efficient electro-optic modulation. Opt. Express 2015, 23, 23072-23078. [CrossRef] [PubMed]

12. Liang, H.; Luo, R.; He, Y.; Jiang, H.; Lin, Q. High-quality lithium niobate photonic crystal nanocavities. Optica 2017, 4, 1251-1258. [CrossRef]

13. Boes, A.; Corcoran, B.; Chang, L.; Bowers, J.; Mitchell, A. Status and potential of lithium niobate on insulator (LNOI) for photonic integrated circuits. Laser Photonics Rev. 2018, 12, 1700256. [CrossRef]

14. Lin, J.; Yao, N.; Hao, Z.; Zhang, J.; Mao, W.; Wang, M.; Chu, W.; Wu, R.; Fang, Z.; Qiao, L. Broadband quasi-phase-matched harmonic generation in an on-chip monocrystalline lithium niobate microdisk resonator. Phys. Rev. Lett. 2019, 122, 173903. [CrossRef] [PubMed] 
15. Zheng, Y.; Fang, Z.; Liu, S.; Cheng, Y.; Chen, X. High-Q exterior whispering-gallery modes in a double-layer crystalline microdisk resonator. Phys. Rev. Lett. 2019, 122, 253902. [CrossRef] [PubMed]

16. Wu, R.; Zhang, J.; Yao, N.; Fang, W.; Qiao, L.; Chai, Z.; Lin, J.; Cheng, Y. Lithium niobate micro-disk resonators of quality factors above $10^{7}$. Opt. Lett. 2018, 43, 4116. [CrossRef] [PubMed]

17. Wu, R.; Wang, M.; Xu, J.; Qi, J.; Chu, W.; Fang, Z.; Zhang, J.; Zhou, J.; Qiao, L.; Chai, Z.; et al. Long low-loss-lithium niobate on insulator waveguides with sub-nanometer surface roughness. Nanomaterials 2018, 8, 910. [CrossRef] [PubMed]

18. Wang, M.; Wu, R.; Lin, J.; Zhang, J.; Fang, Z.; Chai, Z.; Cheng, Y. Chemo-mechanical polish lithography: A pathway to low loss large-scale photonic integration on lithium niobate on insulator. Quantum Eng. 2019, 1, e9. [CrossRef]

19. Fang, Z.; Haque, S.; Lin, J.; Wu, R.; Zhang, J.; Wang, M.; Zhou, J.; Rafa, M.; Lu, T.; Cheng, Y. Real-time electrical tuning of an optical spring on a monolithically integrated ultrahigh $Q$ lithium nibote microresonator. Opt. Lett. 2019, 44, 1214. [CrossRef] [PubMed]

(C) 2019 by the authors. Licensee MDPI, Basel, Switzerland. This article is an open access article distributed under the terms and conditions of the Creative Commons Attribution (CC BY) license (http://creativecommons.org/licenses/by/4.0/). 